\title{
271 Pathophysiology of swallowing
}

A In bulbar palsy swallowing of liquids is characteristically more difficult than solids.

B A vagal paralysis has its main clinical effect on the oesophageal phase of swallowing.

C Cricopharyngeal myotomy is of no benefit if the cause of dysphagia is neurological.

D Globus pharyngis may be associated with gastro-oesophageal reflux.

E Tertiary oesophageal contractions occur frequently during emotional stress.

\section{Stertor}

A Is defined as noisy respiration due to partial airway obstruction below the level of the epiglottis.

$B$ The Venturi effect is implicated in causing the noise.

C The noise is loudest on expiration.

D Intercostal recession is a feature of severe stertor.

E Large tonsils and adenoids may be causative.

\section{Sleep apnoea in children}

A Is a potential cause of sudden infant death syndrome.

$B$ Is associated with failure to thrive and feeding difficulties in the infant.

C Bradycardia is a sign of minor importance.

D A retropharyngeal swelling is best shown on an occipitomental $X$-ray.

E In Pierre-Robin syndrome the respiratory obstruction is most likely to occur in the prone position. 